\title{
The Designation of Human Cardiac Stem Cell Therapy Products for Human Trials
}

\author{
Xuejun H. Parsons ${ }^{1,2 *}$ \\ ${ }^{1}$ San Diego Regenerative Medicine Institute, San Diego, CA 92109 \\ ${ }^{2}$ Xcelthera, San Diego, CA 92109
}

Received: November 26, 2013; Accepted: December 10, 2013, Published: December 16, 2013

${ }^{*}$ Corresponding author: Xuejun H. Parsons, San Diego Regenerative Medicine Institute, USA, Tel: 858-243-2046, E-mail: parsons@SDRMI.org, parsons@xcelthera.com

\section{Editorial}

Cardiovascular disease (CVD) is a major health problem and the leading cause of death in the Western world. About 600,000 people die of heart disease in the United States (US) every yearthat's 1 in every 4 deaths. The estimated costs of CVD for the overall US population are approximately $\$ 190$ billion annually. Currently, there is no treatment option or compound drug of molecular entity that can change the prognosis of CVD. Given the limited capacity of the heart for self-repair or renewal, cell-based therapy represents a promising therapeutic approach closest to provide a cure to restore normal heart tissue and function for CVD. However, traditional sources of cells for therapy in existing markets have been adult stem cells isolated from tissues or artificially reprogrammed from adult cells, which all have the historical shortcomings of limited capacity for renewal and repair, accelerated aging, and immune-rejection following transplantation. In addition, artificially reprogrammed adult cells have the major drawbacks of extremely low efficiencies and genetic defects associated with high risks of cancers, which have severely limited their utility as viable therapeutic approaches. In the adult heart, the mature contracting cardiac muscle cells, known as cardiomyocytes, are terminally differentiated and unable to regenerate. There is no evidence that adult stem/ precursor/progenitor cells derived from mature tissues, such as bone marrow, cord blood, umbilical cord, mesenchymal stem cells, patients' heart tissue, placenta, or fat tissue, are able to give rise to the contractile heart muscle cells following transplantation into the heart [1-7]. To date, the lack of a suitable human cardiomyocyte source with adequate myocardium regenerative potential has been the major setback in regenerating the damaged human heart, either by endogenous cells or by cellbased transplantation or cardiac tissue engineering [1-7]. Despite numerous reports about cell populations expressing stem/ precursor/progenitor cell markers identified in the adult hearts, the minuscule quantities and growing evidences indicating that they are not genuine heart cells and that they give rise predominantly to non-functional smooth muscle cells rather than functional contractile cardiomyocytes have caused skepticism if they can potentially be harnessed for cardiac repair [1-7].

Derivation of human embryonic stem cells (hESCs) from the in vitro fertilization (IVF) leftover embryos has brought a new era of cellular medicine for the heart [1-7]. The intrinsic ability of a hESC to both unlimited self-renew and differentiate into cardiac tissue elements makes it a practically inexhaustible source of replacement cells for the damaged heart. The hESC cardiac cell therapy derivatives are emerging as a new type of pharmacologic agent of cellular entity in cell-based regenerative medicine because they have direct pharmacologic utility and capacity for human myocardial tissue reconstitution and contractile function restoration that the conventional compound drug of molecular entity lacks [1-7]. Although a vast sum of government and private funding has been spent on looking for adult alternates, such as reprogramming and trans-differentiation of fibroblasts or mature tissues, so far, only human cardiac stem/precursor/ progenitor cells derived from embryo-originated hESCs have shown such cellular pharmacologic utility and capacity adequate for myocardium regeneration in pharmaceutical development of stem cell therapy for the damaged heart [1-7].

The pharmacologic activity of human cardiac stem cells is measured by their extraordinary cellular ability to regenerate the functional and structural cardiac tissue element, thus, the contractile cardiomyocytes that has been damaged or lost. Therefore, the pharmacologic utility of human cardiac stem cells cannot be satisfied only by their chaperone activity, if any, to differentiate into non-functional smooth muscle cells or endothelial cells, or produce trophic or protective molecules to rescue endogenous dying cardiomyocytes that can simply be achieved by a compound drug of molecular entity. For successful pharmaceutical development of cardiac stem cell therapy, the human cardiac stem cell therapy product must meet certain commercial criteria in plasticity, specificity, and stability before entry into clinical trials. Moving stem cell research from current studies in animals into human trials must address such practical 
issues for commercial and therapeutic uses: 1) such human stem cells or their cardiac progenies or derivatives must be able to be manufactured in a commercial scale; 2) such human stem cells and their cardiac progenies or derivatives must be able to retain their normality or stability for a long term; and 3) such human stem cells must to be able to differentiate or generate a sufficient number of contractile cardiomyocytes for repair. Those practical issues are essential for designating any human cardiac stem cells as human cardiac stem cell therapy products for INDfiling and entry into clinical trials. On July 9, 2012, the Food and Drug Administration Safety and Innovation Act (FDASIA) was signed into law, which gave FDA a new and powerful expedited drug development tool, known as the "breakthrough therapy" designation. This new designation helps FDA assist drug developers to expedite the development and review of new drugs with preliminary clinical evidence that indicates the drug may offer a substantial improvement over available therapies for patients with serious or life-threatening diseases, such as heart disease and failure. In addition, the FDA has established a Fast Track program that is intended to facilitate the development and expedite the review of new drugs and biological products that are intended to treat a serious or life-threatening condition or disease and demonstrate the potential to address unmet medical needs for the condition. Many of those serious or life-threatening diseases, such as heart disease and failure, have relied on stem cell research to drive the advance of medicine to provide future regeneration and reconstruction treatment options for the damage or lost functional tissues and organs. Because of interspecies differences, conventional preclinical studies using animal models are often poor predictors of human efficacy and safety. Animal models are xeno-hosts for transplantation of human cells, not ideal for testing the safety and efficacy of therapeutic outcomes of human stem cells. Large primate models are very costly and often taken years to obtain results. In addition, the results of animal studies can be highly variable and difficult to reproduce, making them unreliable as benchmarks for decisions on human trials. Preclinical data using animal models, even results of large animal models, do not necessarily provide the benchmarks or indictors for safety and efficacy in human trials. Those FDA expedited programs do not specifically require evidences from animal models or animal proof-of-concept data to support FDA accelerated approval and priority review, which may help fast-track the development and review of human stem cell therapy products that have the potential to provide safe and effective therapy where no satisfactory alternative therapy exists or a significant improvement in the treatment, diagnosis or prevention of a disease compared to the marketed human stem cell therapy product, such as hESC cardiac cell therapy derivatives.

Due to the prevalence of heart disease worldwide and acute shortage of donor organs or adequate human myocardial grafts, there is intense interest in developing hESC-based therapy for heart disease and failure. One of the major challenges in developing hESC therapies for cardiac repair is to determine the necessary molecular and cellular cues that direct efficient cardiac lineage-specific differentiation of pluripotent hESCs. The normal human developmental pathways that generate cardiomyocytes remain poorly understood. As a result, directing hESC differentiation towards cardiomyocytes in a systematic manner has proven difficult. Only a very small fraction of pluripotent hESCs differentiate into cardiomyocytes through spontaneous multi-lineage germ-layer induction in culture [1]. Although such hESC-derived immature cardiomyocytes can be enriched to attenuate the progression of heart failure in acute myocardial infarction model, the grafts generated by cell transplantation have been small and insufficient to restore heart function or to alter adverse remodeling of chronic infarcted models following transplantation [1-7]. Functional enhancement in preclinical animal models by such hESC-derived cardiomyocytes through conventional multi-lineage germ-layer induction has been limited to mid-term at most, equivalent to perhaps a few months in humans, and there is no evidence that the underlying mechanism depends on the contractile properties of the transplanted human cells [1-7]. Growing evidences indicate that incomplete lineage specification of pluripotent cells often result in poor performance of such stem cell derivatives and/or tissue-engineering constructs following transplantation [1-7]. In addition, the need for foreign biologics, such as animal feeder cells and proteins, for derivation, maintenance, and differentiation of hESCs may make direct use of such cells and their derivatives in patients problematic [1-7].

Recent developments in hESC research have overcome some major obstacles in moving stem cell research from current studies in animals towards humans for clinical trials, including resolving minimal essential human requirements for de novo derivation and long-term maintenance of clinically-suitable stable hESC lines and direct conversion of such pluripotent hESCs into a large supply of clinical-grade functional human neuronal or cardiomyocyte cell therapy products to be translated to patients for CNS or heart repair [1-15]. Without an understanding of the essential developmental components for sustaining hESC pluripotence and self-renewal, hESC lines are at risk for becoming unhealthy and unstable after prolonged culturing under animal feeders, feeder-conditioned media, or artificially-formulated chemically-defined conditions [1, 6-8]. Resolving minimal essential human requirements for sustaining embryonic pluripotence allows all poorly-characterized and unspecified biological components and substrates in the culture system, including those derived from animals, to be removed, substituted, and optimized with defined human alternatives for de novo derivation and long-term maintenance of GMP-quality xeno-free stable hESC lines and their human stem cell therapy derivatives [1, 6-8]. Formulation of minimal essential defined conditions renders pluripotent hESCs be directly and uniformly converted into a specific neural or cardiac lineage by small signal molecule induction [1-15]. Nicotinamide (NAM) was identified sufficient to induce the specification of cardiomesoderm direct from the pluripotent state of hESCs maintained under the defined culture, without going through a multi-lineage embryoid body 
stage, by promoting the expression of the earliest cardiac-specific transcription factor $\mathrm{Csx} / \mathrm{Nkx} 2.5$ and triggering progression to cardiac precursors and beating cardiomyocytes with high efficiency $[1,6-8,10,12]$. Cells within the beating cardiospheres expressed markers characteristic of cardiomyocytes, including Nkx2.5, GATA-4, $\alpha$-actinin, cardiac troponin I (cTnI), and cardiac troponin $\mathrm{T}$ (cTnT) $[1,6-8,10,12]$. Electrical profiles of the cardiomyocytes confirmed their contractions to be strong rhythmic impulses reminiscent of the p-QRS-T-complexes seen from body surface electrodes in clinical electrocardiograms [6-8,10]. Our novel approach of hESC cardiac lineage-specific differentiation direct from the pluripotent stage using small molecule induction is a major milestone towards human trials of hESC cardiac cell therapy derivatives, offering the benefits in efficiency, purity, stability, safety, and scale-up production of clinical-grade hESC cardiac cell therapy products in cGMP facility over all other existing conventional approaches [1-7]. Currently, these hESC cardiomyocyte cell therapy derivatives or products are the only available human cell sources in large commercial scales with adequate utility and capacity to regenerate the contractile heart muscles, vital for heart repair in the clinical setting [1-7]. The availability of human heart precursors and cardiomyocytes in high purity and large commercial scales with adequate pharmacologic capacity for myocardium regeneration will expedite moving stem cell research from animals to human trials, leading to FDA approval of hESC cardiac cell therapy products as a treatment or cure for heart disease and failure that currently affect millions of survivors and where so far no satisfactory alternative therapy exists or a significant improvement in the treatment.

\section{References}

1. Parsons, X. H., Teng, Y. D., Moore, D. A., \& Snyder, E. Y. (2011). Patents on technologies of human tissue and organ regeneration from pluripotenthESCs. Recent Patents on Regenerative Medicine, 1(2), 142-163.

2. Parsons, X. H. (2012). Mending the broken heart - Towards clinical application of human embryonic stem cell therapy derivatives. Journal of Clinical \& Experimental Cardiology, 3(12).

3. Parsons, X. H. (2013). Cellular medicine for the heart - the pharmacologic utility and capacity of human cardiac stem cells. Journal of Clinical \& Experimental Cardiology, S11-e001.
4. Parsons, X. H. (2013). Exploring future cardiovascular medicine: heart precursors directed from human embryonic stem cells for myocardium regeneration. Cardiol Pharmacol, 2(3).

5. Parsons, X. H. (2013). Reviving cell-based regenerative medicine for heart reconstitution with efficiency in deriving cardiac elements from pluripotent human embryonic stem cells. Cardio Pharmacol, 2(3).

6. Parsons, X. H. (2013). Constraining the pluripotent fate of human embryonic stem cells (hESCs) for tissue engineering and cell therapy The turning point of cell-based regenerative medicine. British Biotech Journal, 3(4), 424-457.

7. Parsons, X. H. (2013). Embedding the future of regenerative medicine into the open epigenomic landscape of pluripotent human embryonic stem cells. Annual Review \& Research in Biology, 3(4), 323-349.

8. Parsons, J. F., Smotrich, D. B., Gonzalez, R., Snyder, E. Y., Moore. D. A., \& Parsons, X. H. (2012). Defining conditions for sustaining epiblast pluripotence enables direct induction of clinically-suitable human myocardial grafts from biologics-free human embryonic stem cells. Journal of Clinical \& Experimental Cardiology, S9-001.

9. Parsons, X. H., (2012). The dynamics of global chromatin remodeling are pivotal for tracking the normal pluripotency of human embryonic stem cells. Anatomy and Physiology, S3, 002.

10. Parsons, X. H., Teng, Y. D., Parsons, J. F., Snyder, E. Y., Smotrich, D. B., \& Moore, D. A.(2011). Efficient derivation of human cardiac precursors and cardiomyocytes from pluripotent human embryonic stem cells with small molecule induction. Journal of visualized experiments, 57.

11. Parsons, X. H., Teng, Y. D., Parsons. J. F., Snyder, E.Y., Smotrich, D. B., \& Moore, D. A. (2011). Efficient derivation of human neuronal progenitors and neurons from pluripotent human embryonic stem cells with small molecule induction. Journal of visualized experiments, 56.

12. Parsons, X. H. (2012). MicroRNA profiling reveals distinct mechanisms governing cardiac and neural lineage-specification of pluripotent human embryonic stem cells. Stem Cell Research \& Therapy, 2(3), 124.

13. Parsons, X. H., Parsons, J. F., \& Moore, D. A. (2013). Genome-scale mapping of microRNA signatures in human embryonic stem cell neurogenesis. Molecular Medicine \& Therapeutics, 1(2).

14.Parsons, X. H. (2012). An engraftable human embryonic stem cell neuronal lineage-specific derivative retains embryonic chromatin plasticity for scale-up CNS regeneration. Journal of Tissue Engineering and Regenerative Medicine, 1(3).

15. Parsons, X. H. (2013). Human stem cell derivatives retain more open epigenomic landscape when derived from pluripotent cells than from tissues. Journal of Regenerative Medicine, 1(2). 